\title{
ABSTRACTS
}

The Keio Medical Science Prize 2019 Commemorative Lectures

\section{Organoids: Avatars for Personalized Medicine}

\author{
Hans C. Clevers \\ Molecular Genetics, University Medical Center Utrecht, Utrecht, the Netherlands \\ Hubrecht Institute of the Royal Netherlands Academy, Utrecht, the Netherlands \\ Princess Máxima Center for Pediatric Oncology, Utrecht, the Netherlands
}

Stem cells are the foundation of all mammalian life. Stem cells build and maintain our bodies throughout life. Two types of stem cells are discerned.

1) Embryonic stem cells (ES cells) are briefly present in the early human or mouse embryo, a few days after fertilization. These ES cells can be grown indefinitely in the lab and have the potential to build each and every tissue in our body. Because of this 'pluripotency', ES cells hold great promise for therapeutic application in the field of regenerative medicine. It is also possible to take skin cells (or other cells) from adults and convert these in the lab into cells with ES properties, so called iPS cells. Many of the hurdles that ES cell technology have faced, do not exist for iPS cells.

2) Adult stem cells. Every organ in our body is believed to harbor its own dedicated stem cells. These adult stem cells replace tissue that is lost due to wear and tear, trauma and disease. Adult stem cells are highly specialized and can only produce the tissue in which they reside; they are 'multipotent'. Examples are bone marrow stem cells that make all blood cells, skin stem cells and gut stem cells. Even the brain is now known to harbor its specialized stem cells. The adult stem cells allow us to live 8090 years, but this comes at a cost: they are the cells that most easily transform into cancer cells.

Both types of stem cells can be used to establish 'organoids', 3D structures established in a dish, that recapitulate many aspects of the organ they represent. Pluripotent stem cells can be taken through the developmental steps that establish organs during embryogenesis. This has worked particularly well for parts of the the central nervous system, the kidney and GI organs. We have shown that adult epithelial stem cells carrying the generic Lgr5 marker can be cultured under tissue-repair conditions and generate epithelial organoids directly from healthy and diseased organs such as the gut, the liver, the lung and the pancreas. Organoid technology opens a range of avenues for the study of development, physiology and disease, for drug development and for personalized medicine. In the long run, cultured mini-organs may replace transplant organs from donors and hold promise in gene therapy. 